\title{
Aprendizaje cooperativo intergrados para la potenciación de distintos perfiles profesionales: creación de un glosario de LFE*
}

Leticia Moreno Pérez, ${ }^{\text {a }}$ y Belén López Arroyo ${ }^{b}$

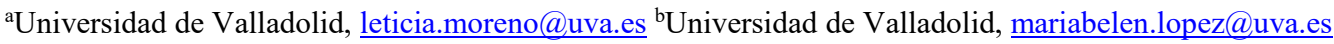

\section{\$EWWFW}

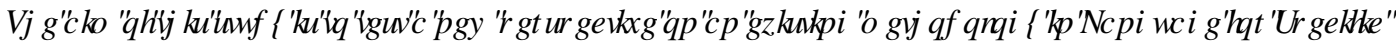

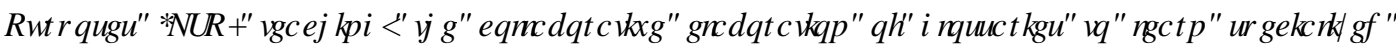

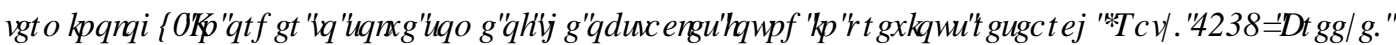

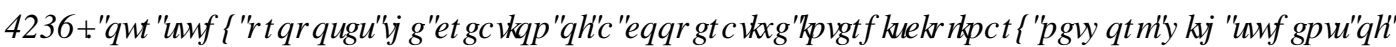

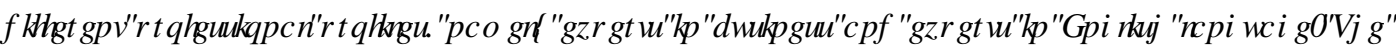

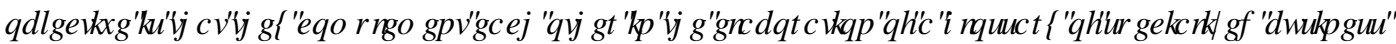

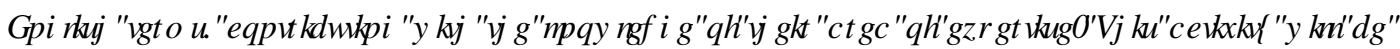

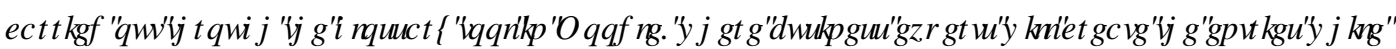

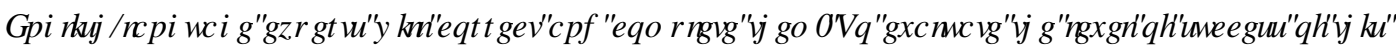

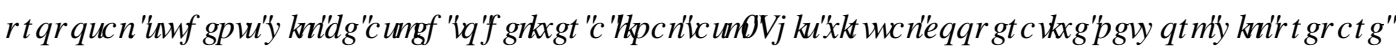

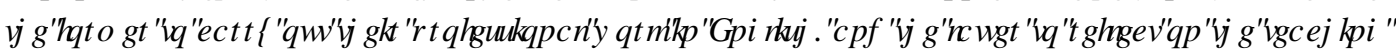

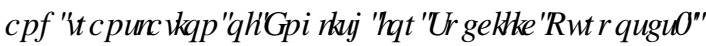

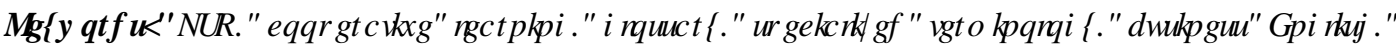
ISHFDO] HOWWDIDURQ

प

\section{HXP HQ}

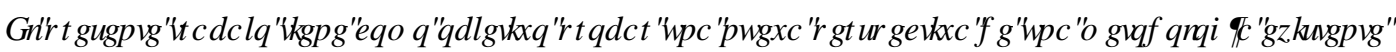

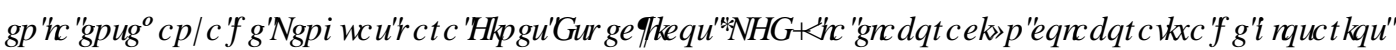

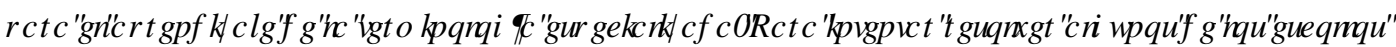

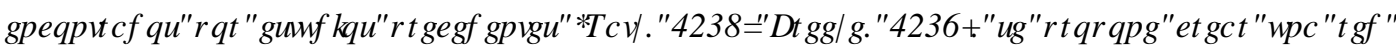

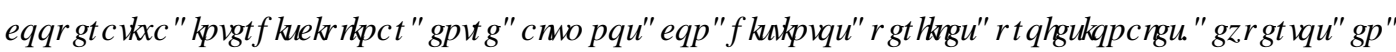

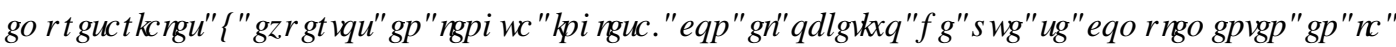

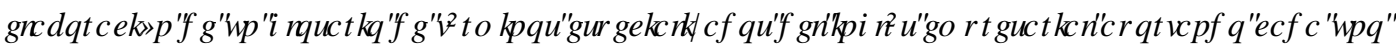
GRV FRQRFIP IHQURV SLRSIRV GHWW

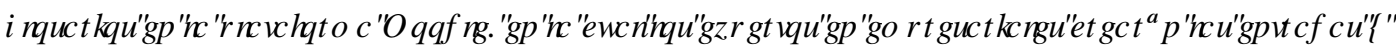

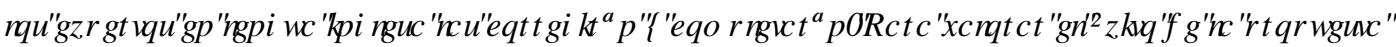

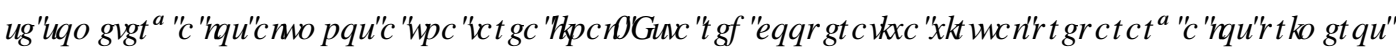

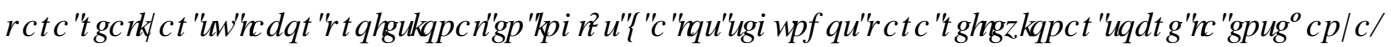

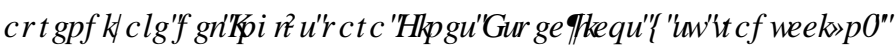

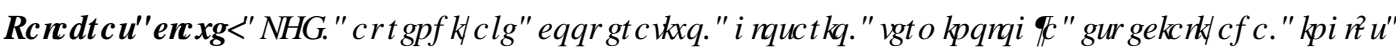
H SUMDUDOWDOXFFIy QHSHFD] DOD]

\footnotetext{
* Este trabajo se enmarca dentro del Proyecto de Innovación Docente "BusinessCom" aprobado por la Universidad de Valladolid en su convocatoria 2020/2021
} 


\section{Introducción}

La tendencia hacia la especialización de la enseñanza superior ha traído, entre otras consecuencias, un aumento en la necesidad de formar a los futuros profesionales para que puedan desenvolverse en sus empleos en otros idiomas. Estas lenguas profesionales, que suponen la base de la comunicación dentro de los sectores y ámbitos de especialidad propios del mundo laboral, son lo que se denomina Lenguas para Fines Específicos (LFE). Se diferencian principalmente de la lengua general en que suelen girar en torno a "[...] una temática específica, utilizada en situaciones pragmáticas determinadas" (Aguado, 2002: 18), y entre sus principales características destacan el uso de una terminología especializada para denominar los conceptos dentro de cada disciplina profesional (Cabré, 2005), el recurso a elementos sintácticogramaticales distintivos que se apartan de la norma habitual o la existencia de una serie de géneros textuales concretos propios de cada área de especialidad que se emplean para la comunicación en los distintos tipos de situaciones de ese ámbito profesional.

De todos estos elementos distintivos, "la terminología es sin duda el escollo más inmediato con el que se encontrará cualquier aprendiz de una LFE" (Moreno-Pérez, 2016: 64), motivo por el cual este será uno de los puntos clave en el aprendizaje de cualquier lengua extranjera de especialidad, más concretamente porque en esta área de la enseñanza de idiomas uno de los objetivos es que los aprendices se conviertan en usuarios de dicha lengua de forma prácticamente inmediata (Carver, 1983: 134). Por ello, es necesario recurrir a técnicas docentes específicas de esta disciplina, las cuales se adapten a las necesidades profesionales que tienen o tendrán los aprendices. Entre estas técnicas destacan la simulación de situaciones laborales en el aula o la resolución de estudios de caso dentro del contexto profesional (Rodríguez y García, 2009). Si nos centramos en la enseñanza-aprendizaje concreto de la terminología encontramos referencias en la literatura a metodologías como el empleo de corpus para la extracción y recopilación de términos, o la elaboración de glosarios a modo de base de datos terminológica. Esta última metodología ha probado en estudios previos resultar de gran utilidad para el aprendizaje de vocabulario en lenguas extranjeras, y es por ello que será el objeto de estudio del presente trabajo.

Ratz (2016) propuso en su estudio la elaboración de un glosario cooperativo como tarea para el aprendizaje de vocabulario en el aula de idiomas (lengua general), y observó que su elaboración tuvo un efecto positivo en el desempeño de los estudiantes en las actividades de clase, además de ser percibido como útil por los propios alumnos. Esta metodología, resultados y percepción de los alumnos se ha reflejado también en la enseñanza-aprendizaje de LFE, como demuestran los estudios de Pospíšilová, Bezdíčková y Ciberová (2011) en el área de la química o de Breeze (2014) en el lenguaje jurídico. Por último, y también relacionado con las LFE, existen estudios en la misma línea en el ámbito de la traducción especializada, como el de Seghiri (2013), centrado en la creación de un glosario para la traducción de textos científico-técnicos y de gran utilidad para el aprendizaje y consulta de los futuros traductores.

Observamos que estas experiencias previas no solo tienen en común los resultados positivos para la adquisición del vocabulario en otra lengua, sino que la mayoría se han desarrollado de forma cooperativa entre los estudiantes de un curso o asignatura a través de plataformas de aprendizaje, más concretamente Moodle. Esto refleja una realidad imperante en los modelos educativos actuales, que giran en torno a la adquisición de destrezas en el mundo digital y "al concepto de cooperación como motor de la enseñanza y el aprendizaje” (Alarcón, Sepúlveda y Madrid, 2018: 210). Estas metodologías basadas en el aprendizaje cooperativo, por lo general, requieren que el docente sea quien dirija y estructure claramente la realización de las tareas, y tienen especial valor para alumnos con niveles más bajos (Alarcón, Sepúlveda y Madrid, 2018: 212), lo cual es especialmente significativo en el aula de LFE, al darse habitualmente la situación de 
que los discentes suelen tener un nivel limitado de partida en la lengua extranjera en general (Laborda y Litzler, 2015: 42).

A pesar de los resultados positivos, los estudios previos también apuntan a una serie de aspectos negativos en la aplicación de la metodología de la elaboración cooperativa de glosarios. A parte de pérdida de motivación por parte de los alumnos si la tarea se desarrolla durante un periodo dilatado o el hecho de que el trabajo cooperativo y en entornos digitales no son las metodologías predilectas de todos los estudiantes (Ratz, 2016), quizás el principal escollo que se ha observado es el hecho de que, al estar realizando un glosario entre pares, los estudiantes tienen dudas sobre la idoneidad del trabajo de sus compañeros de clase, pues no son expertos en la lengua de trabajo del glosario y dependen siempre de las correcciones del profesor (Breeze, 2014).

Dada la utilidad de la metodología expuesta consideramos relevante buscar modos de pulirla para hacer que sea más eficiente y efectiva.

\section{Objetivos}

El presente trabajo tiene como objetivo aportar un nuevo punto de vista a la metodología de enseñanzaaprendizaje de terminología de LFE basada en la elaboración cooperativa de un glosario. Con intención de intentar solventar las principales deficiencias de esta metodología detectadas en las investigaciones previas, la innovación propuesta en el presente estudio implica cambiar el perfil de los estudiantes involucrados: no se elaborará el glosario exclusivamente entre compañeros de clase del mismo curso y estudios (concretamente, expertos en el mundo empresarial que están aprendiendo inglés de ese área), sino que se elaborará en cooperación con estudiantes de otro perfil profesional (concretamente, expertos en lengua inglesa que están aprendiendo conceptos empresariales para la traducción), de modo que complementen mutuamente sus conocimientos. El fin último es que los estudiantes se sientan motivados al actuar como expertos en sus campos de conocimiento a la vez que podrán nutrirse de los conocimientos de los otros, permitiendo la actividad reforzar varios perfiles al mismo tiempo, cada uno para sus intereses.

El empleo de la figura del experto en otro campo como apoyo para la realización y optimización del trabajo propio es una idea avalada especialmente por los Estudios de Traducción. Debido a las características propias del mercado de la traducción, los profesionales de este sector se ven habitualmente obligados a trabajar en distintos campos de especialidad sin centrarse ni acabar de especializarse totalmente en uno; a pesar de ello, el cliente espera que el resultado de sus traducciones cumpla las convenciones propias del campo en que se encuadre como si hubiera sido redactado por un experto (Moreno-Pérez y López-Arroyo, en prensa). Es por esto que el trabajo mano a mano del traductor con un experto del campo en que tenga que traducir se convierte en "una fuente de documentación privilegiada" (Alarcón, 2010: 221), y que la cooperación entre ambos profesionales sea considerada como "una de las herramientas más útiles para lograr traducciones aceptables entre el colectivo al que van dirigidas" (Muñoz-Miquel, 2015: 336).

En su estudio, esta última autora realizó un experimento en el que promovió el trabajo cooperativo entre traductores en formación y expertos. Se observó que los dos motivos principales por los que se solicitaba la ayuda de los expertos era, en primer lugar, para seleccionar el equivalente terminológico adecuado en el área especializada y en segundo lugar, para que el experto revisara la adecuación de alguna "frase o párrafo ya traducido" (Muñoz-Miquel, 2015: 342). A nuestro juicio, consideramos que esta misma dinámica se podría extrapolar a la situación propuesta para nuestra investigación, pues los expertos en empresariales pueden ayudar a los expertos lengua inglesa con la precisión y adecuación de los equivalentes terminológicos, mientras que los segundos pueden ayudar a los primeros en la revisión de sus propuestas de redacción a la hora de crear ejemplos de uso en el glosario en lengua inglesa. En este contexto, el profesor

(c)) EY-NC-ND 2021, Universitat Politècnica de València

CRQJUHRL, Q5 HGस००स० 
actuará como coordinador y revisor último del trabajo de los estudiantes, al tratarse de expertos en formación, por lo que "habrá que ser cuidadoso con sus respuestas debido a que aún puede[n] no tener afianzadas dichas competencias y conocimientos" (Huertas, 2013: 83). De este modo, la innovación propuesta pretende que el perfil de expertos en empresariales no solo aprendan la terminología de su LFE en lengua inglesa sino que también mejoren el uso de la lengua. Al mismo tiempo, se estaría logrando que los expertos en lengua inglesa, con un perfil multidisciplinar entre la docencia y la traducción, no solo aprendan la terminología y conceptos de esta LFE para enfrentarse a la traducción de sus textos, sino también a su enseñanza mediante el análisis y corrección de los fallos de sus compañeros.

\section{Desarrollo de la innovación}

\subsection{Perfiles}

El estudio aquí planteado consta de alumnos de dos perfiles definidos por su papel en la tarea, cada uno de ellos con unas necesidades específicas:

a) Expertos en empresariales: estudiantes del Grado en Comercio de segundo curso, alumnos de la asignatura Lengua Extranjera Inglés II con un nivel aproximado B1-B2 de lengua general y conocimientos en materias como economía, derecho, organización de empresas o marketing. Sus necesidades son aprender el lenguaje de especialidad del comercio y el mundo de la empresa en inglés a nivel terminológico y sintáctico-gramatical.

b) Expertos en lengua inglesa: estudiantes del Grado en Estudios Ingleses de tercer curso, alumnos de la asignatura Traducción de Lenguajes de Especialidad I (inglés-español), centrada en la introducción a la traducción de textos jurídico-económicos y con conocimientos de lengua inglesa a nivel gramática, técnicas de expresión escrita o teoría y práctica de la traducción. Sus necesidades son aprender los conceptos y términos propios del lenguaje jurídico-económico.

\subsection{Fases de trabajo}

El desarrollo de la actividad se planteó en varias fases de trabajo que fueran compatibles con el desarrollo de las asignaturas de ambos grupos y con la temporalización del curso.

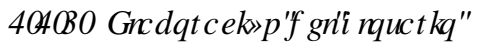

La primera fase consistió en la presentación del proyecto a los alumnos: para los alumnos de Comercio se presentó como una metodología innovadora que les ayudaría a sintetizar mejor el vocabulario especializado, resultándoles relevante al coincidir la mayoría de ellos en la poca eficiencia que habían tenido las metodologías a las que se habían expuesto anteriormente en asignaturas de idiomas. Para los alumnos de Estudios Ingleses supondría una oportunidad para aprender conceptos y terminología del mundo empresarial para ayudarles a enfrentarse a la traducción de textos especializados en esa área, además de para acercarles a la docencia de LFE en su labor como revisores y correctores del glosario. En ambos casos, la tarea se calificó con un porcentaje en la evaluación final.

Comenzaron trabajando los alumnos de Comercio, a quienes se les presentó la herramienta de elaboración de glosarios en la plataforma de apoyo a la docencia online Moodle y se les proporcionaron unas pautas sobre su elaboración: el glosario constaría del término en inglés, categoría gramatical, equivalente en español, oración ejemplo de uso en inglés y campo temático en que se emplea (por ejemplo, marketing), 
dependientes éstos de las unidades en que se divide la asignatura. Se les concedió al inicio un periodo de práctica para que introdujeran varias palabras en una categoría general y poderles ir corrigiendo los fallos, los cuales se marcaron no solo en el propio glosario para que todos pudieran visualizarlos, sino que se pusieron en común y explicaron en una clase magistral. A partir de ese momento, y durante 4 unidades, los alumnos fueron elaborando de forma autónoma las entradas del glosario con los términos más relevantes indicados por el profesor. Semanalmente ciertos alumnos concretos tenían la tarea de subir los términos que se les solicitaba al glosario común, para evitar multiplicidad de entradas.

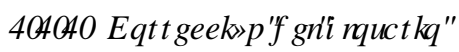

Si bien la idea inicial era que los alumnos de Estudios Ingleses fueran corrigiendo gradualmente las entradas del glosario, no fue posible seguir esta pauta por la diferente temporalización de ambas asignaturas, así como ciertas restricciones espacio-temporales surgidas a causa de la nueva organización de la docencia en situación de nueva normalidad tras la pandemia de la COVID-19. Por estos motivos, los alumnos realizaron las correcciones una vez concluida la fase de elaboración del glosario. Se repartió el número total de entradas del glosario de forma equitativa entre los alumnos participantes, a quienes se les solicitó que revisaran cada una de ellas y realizaran los cambios que consideraran necesarios para mejorarlas tanto a nivel lingüístico como terminológico (adecuación del término y el equivalente, adecuación de término y ejemplo, adecuación de la categoría gramatical, corrección de errores sintáctico-gramaticales en los ejemplos de uso...). Para que pudieran aprovechar al máximo la tarea en lo que respecta al aprendizaje de conceptos y términos especializados, se les solicitó que, una vez corregidas, incluyeran una oración ejemplo de uso de cada término tanto en inglés como en español.

Una vez finalizadas, entregaron las correcciones y sus aportaciones al profesor, quien las revisó e introdujo la versión final en Moodle para su consulta y descarga por parte de los alumnos de Comercio, quienes desde ese momento tuvieron a su disposición la herramienta completa para emplearla en las tareas restantes de la asignatura.

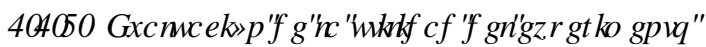

Para probar el grado de éxito de la tarea y su utilidad para los alumnos se llevaron a cabo dos evaluaciones diferenciadas por perfil:

a) Expertos en empresariales: tarea final escrita compendio de todo lo trabajado en la asignatura en la que tenían que redactar un texto especializado del mundo empresarial en inglés, concretamente un informe, siguiendo unas instrucciones en un contexto dado que requería que demostraran su manejo del vocabulario especializado.

b) Expertos en lengua inglesa: encuesta de valoración en la que se pedía a los alumnos que dieran su opinión sobre las correcciones a nivel lingüístico (reflexión sobre la enseñanza-aprendizaje de LFE en base a los errores que encontraron) y a nivel traductológico (análisis sobre fuentes consultadas y el proceso de toma de decisiones), además de una valoración final sobre cómo podría ayudar la tarea en su futuro como profesionales de la lengua inglesa. 


\section{Resultados}

\subsection{Nivel de éxito de la tarea para los expertos en empresariales}

Para evaluar la influencia de la tarea en el aprendizaje y manejo del vocabulario especializado se requirió la misma actividad final de redacción a otro grupo de alumnos de la misma asignatura pero que no habían realizado la tarea del glosario. De este modo, el grupo de alumnos que elaboró el glosario actuó como grupo experimental, mientras que los que no lo realizaron sirvieron como grupo de control para contrastar los resultados.

Se tuvieron en cuenta tres variables generales a la hora de analizar los resultados: la densidad terminológica, es decir, el número total de términos especializados del lenguaje empresarial empleado; la corrección en el uso de los términos empleados; y la naturaleza de los errores cometidos.

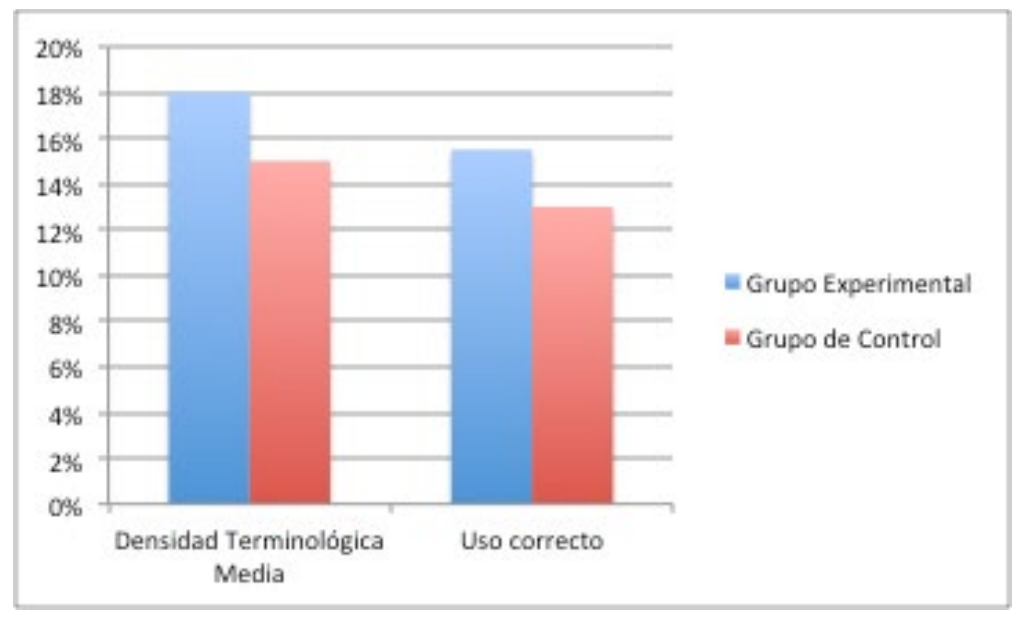

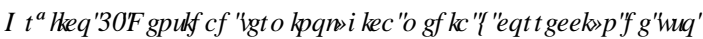

a) Densidad terminológica (Gráfico 1): el grupo experimental muestra una densidad terminológica media del 18\% frente al 15\% del grupo de control. Si bien no se trata de porcentajes demasiado elevados en el uso de términos, es importante tener en cuenta que el texto que tenían que producir se podría categorizar como semiespecializado (Rodríguez-Tapia, 2016), por lo que es un rango de densidad terminológica razonable, más aún cuando se trata de términos que los alumnos acaban de aprender. Esta media de un 3\% superior del grupo experimental se ve refrendada, además, si se analiza individualmente la media de cada alumno: los alumnos con un porcentaje más alto de empleo de términos especializados a nivel individual se encuentran, también, en el grupo experimental, quienes emplean un $6,25 \%$ más de términos propios del mundo empresarial que sus compañeros del grupo de control. Consideramos importante en este punto destacar que, antes de que los alumnos realizaran la tarea final, se realizó con todos (ambos grupos) un trabajo terminológico específico con unos términos muy concretos del contexto en que se encuadraba dicha tarea. Si se analiza la terminología empleada por los dos grupos podemos observar que el grupo de control utiliza muchos más términos de los trabajados de forma específica inmediatamente antes de la actividad frente al grupo experimental, que emplea más términos empresariales de otros tipos. Esto también parece apoyar los hallazgos anteriores.

b) Corrección (Gráfico 1): de media, el grupo experimental tiene un mejor desempeño en el uso de los términos, pues del $18 \%$ de términos especializados empleados, un $15,5 \%$ se emplearon correctamente, 
frente al 13\% de términos empleados correctamente sobre el 15\% de media total del grupo de control: esto supone un desempeño mejor por parte del grupo experimental del $2,5 \%$.

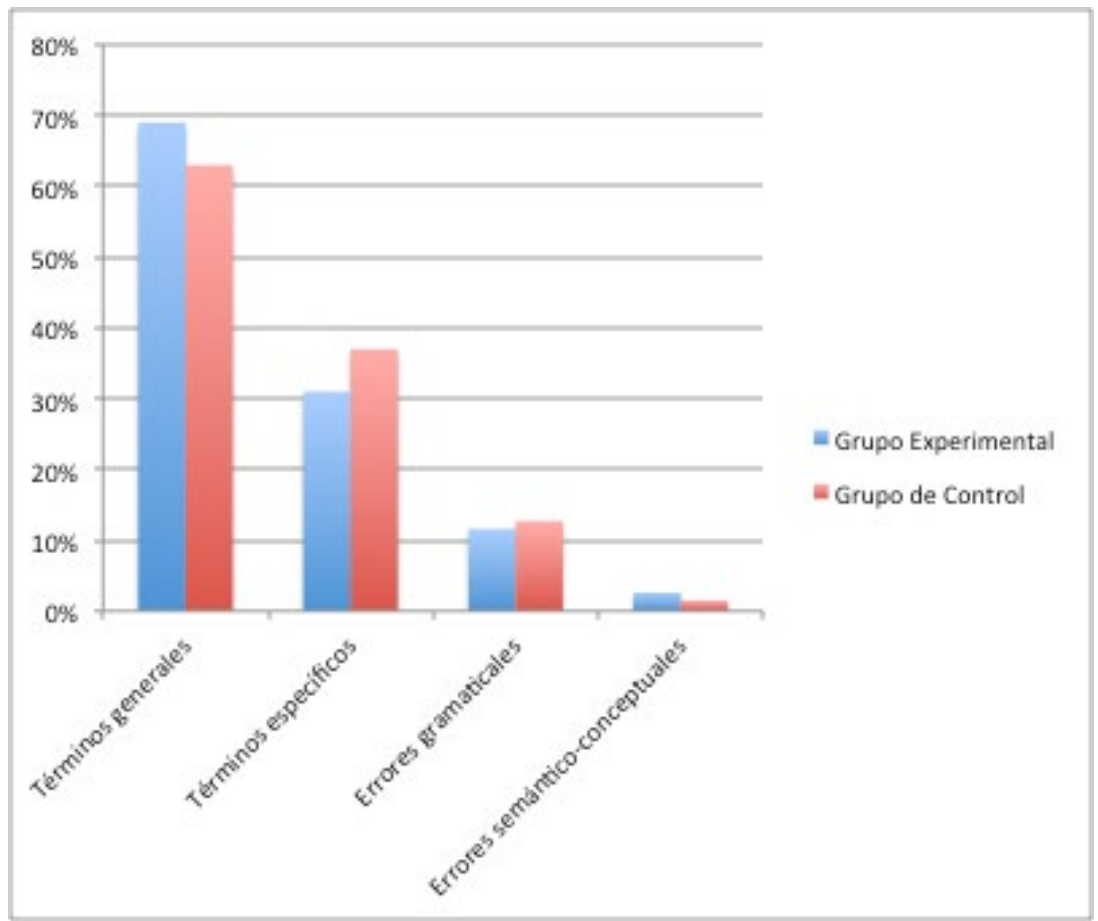

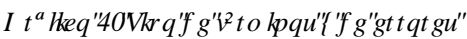

c) Tipología de errores (Gráfico 2): para evaluar esta categoría se asignaron los errores a dos categorías, errores por uso incorrecto a nivel gramatical (errores de escritura, errores relacionados con mal empleo de los plurales, verbos mal conjugados, etc.) y errores por uso incorrecto a nivel semánticoconceptual (confusión de palabras con diferente significado, confusión de categoría gramatical que supone un cambio de significado, etc.). Observamos que ambos grupos emplean incorrectamente el $14,2 \%$ de los términos, siendo los errores gramaticales los más habituales (11,6\% en el grupo experimental, $12,7 \%$ en el grupo de control). Los errores semántico-conceptuales suponen un porcentaje proporcionalmente bajo en ambos casos, pero es superior en el grupo experimental $(2,6 \%)$ frente al grupo de control $(1,5 \%)$. Esto se debe, seguramente, a que los errores semántico-conceptuales se han detectado exclusivamente en el empleo de términos generales del mundo empresarial (empleados mayormente por el grupo experimental), mientras que los errores gramaticales se cometen tanto en los términos generales como en los específicos de la tarea.

En términos generales, los resultados analizados apuntan a un mejor desempeño por parte del grupo experimental en todas las categorías.

\subsection{Nivel de éxito de la tarea para los expertos en lengua inglesa}

Para evaluar la utilidad para alumnos de Estudios Ingleses se les realizó una encuesta con el fin de recoger datos objetivos y subjetivos de su parte de la tarea; se pretendía así valorar la utilidad de la actividad para potenciar tanto el perfil docente de los participantes (parte de corrección gramatical del glosario) como el perfil traductor (parte de corrección terminológica y parte de compleción). 
a) Potenciación del perfil docente: en la parte objetiva destinada a analizar la utilidad para el perfil docente, los alumnos debían recoger el tipo de errores que habían encontrado en las entradas del glosario, coincidiendo todos ellos en la corrección de errores gramaticales, fundamentalmente, así como algunos errores terminológicos. El objetivo de solicitar que resumieran sus correcciones era que reflexionaran sobre las necesidades de aprendizaje de los alumnos con ese nivel de dominio lingüístico, reflexiones que se recogieron en la parte subjetiva. Según sus comentarios, los alumnos especialistas en lengua inglesa se vieron sorprendidos por el tipo de errores encontrados, ya que no los esperaban en estudiantes de este nivel, y manifestaron la utilidad que esta actividad había tenido para ser conscientes de las carencias y necesidades en este estadio del aprendizaje, algo a lo que en su mayoría no habían tenido oportunidad de enfrentarse con anterioridad.

b) Potenciación del perfil traductor: en la parte objetiva destinada a analizar la utilidad para el perfil traductor, los alumnos debían especificar las fuentes y el proceso que habían seguido para revisar los equivalentes terminológicos y elegir los ejemplos de uso. La mayoría de ellos emplearon diccionarios generales, textos paralelos y corpus en línea, herramientas con las que se les enseña a trabajar en la asignatura de traducción en cuestión. En la parte subjetiva el objetivo era que reflexionaran sobre las necesidades terminológicas y conceptuales que los alumnos percibían que podían tener en su labor como traductores de textos especializados y sobre cómo esta herramienta y actividad les podía ayudar. En sus respuestas manifestaron que la actividad les ha resultado interesante para darse cuenta de la necesidad de tener un buen conocimiento enciclopédico en este tipo de traducción, y sugirieron posibles mejoras para que la herramienta resultante, el glosario, les resultara más útil en esta labor, como la adición de las categorías de campo/subcampo de especialidad y la inclusión de las definiciones de los términos, transformándolo en un diccionario.

Los resultados analizados para este perfil también resultan positivos, fomentando tanto la potenciación del perfil docente como del perfil traductor.

\section{Conclusiones}

En términos generales, la actividad de elaboración y corrección del glosario de términos del mundo empresarial parece haber resultado de utilidad para mejorar el desempeño de ambos perfiles de estudiantes: los alumnos expertos en empresariales muestran una mayor seguridad en el manejo del vocabulario especializado de la asignatura, mientras que los alumnos expertos en lengua inglesa han aprendido aspectos relacionados con las necesidades docentes de los estudiantes de nivel B1-B2 y tienen una nueva base de datos terminológica de esta área de especialidad. A la luz de los resultados obtenidos, las principales conclusiones del presente estudio para ambos perfiles son las siguientes:

- Los datos de la densidad terminológica confirman que, a pesar de las inseguridades que todavía puedan sentir los alumnos de Comercio a la hora de emplear el vocabulario especializado del mundo empresarial que acaban de aprender, el hecho de haber seguido un proceso de recopilación y trabajo sobre los términos para elaborar el glosario parece proporcionarles más confianza a la hora de emplearlos. Esta mayor variedad de términos también implica que cometen más variedad de errores.

- Los datos obtenidos nos permiten observar que prácticamente el $90 \%$ de los términos que emplean los alumnos de Comercio se utilizan de forma correcta. Por tanto, parece que los alumnos de Comercio adquieren de forma adecuada la terminología de la asignatura en términos generales. En cualquier caso, sí que es digno de mención que el porcentaje de éxito del grupo experimental sigue 
siendo ligeramente superior, lo que puede ser reflejo de la mayor exposición y trabajo que se han tenido estos alumnos forma específica con los términos al elaborar el glosario.

- Consideramos probada la utilidad de la tarea para los alumnos de Estudios Ingleses en cuanto a la potenciación de su perfil docente al manifestar los encuestados que anteriormente no habían tenido la oportunidad de reflexionar sobre las necesidades docentes de alumnos de estos niveles, lo cual es esencial en el aula de idiomas.

- En la parte traductora, los alumnos de Estudios Ingleses demuestran también aplicar los conocimientos adquiridos en la asignatura, a pesar de que no realizan una distinción clara entre glosario y diccionario, en lo cual se deberá incidir en su formación.

A pesar de que los resultados parecen apuntar a una mejora en prácticamente todos los aspectos propuestos, consideramos necesario trabajar para incrementar los porcentajes de éxito mostrados en este estudio para los alumnos especialistas en empresariales, así como para producir una herramienta más completa y comprobar de forma empírica cómo mejora el rendimiento de los alumnos especialistas en lengua inglesa en su labor traductora.

Ya se está trabajando en una réplica de este experimento con ciertas mejoras implementadas para intentar alcanzar estos objetivos: por un lado, se reforzará la tarea de elaboración del glosario con actividades específicas de vocabulario para los alumnos de Comercio, de modo que sinteticen mejor la terminología. Además, si las circunstancias lo permiten, se intentará sincronizar elaboración y correcciones para que se sucedan de manera prácticamente simultánea y los alumnos puedan practicar sobre ese feedback. Por otro lado, se incluirán en la nueva versión el campo/subcampo de especialidad, pues puede ser de utilidad para ambos perfiles a la hora de manejar el vocabulario; de momento no se plantea la opción de transformar el glosario en diccionario, pero se estudiará el modo de hacerlo en el futuro. Por último, una vez terminada la tarea se propondrá una actividad de traducción especializada a los alumnos de Estudios Ingleses para comprobar la efectividad del uso del glosario y de su corrección.

Esperamos haber contribuido con este estudio a la mejora de la metodología propuesta y consideramos que los resultados nos van a permitir continuar buscando el modo de sacarle aún más provecho en el futuro.

\section{Referencias}

AGUADO, G. (2002). "Lenguas para fines específicos y terminología: algunos aspectos teóricos y prácticos" en Bravo,

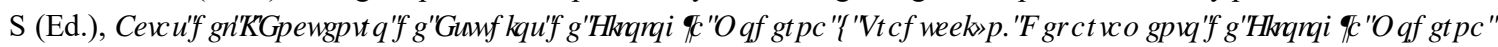

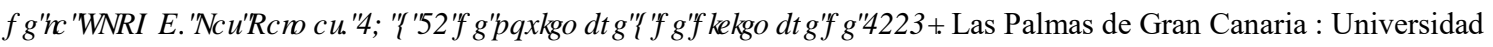
de Las Palmas de Gran Canaria. P. 15-27.

ALARCÓN NAVÍO, E. (2010) "La consulta a expertos como recurso didáctico en la formación del traductor científico

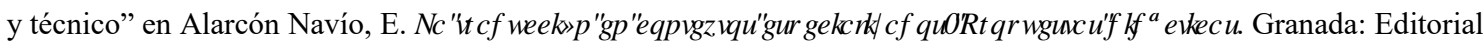
Atrio. P. 219-233.

ALARCON, E., SEPULVEDA, P. y MADRID, D. (2018). "Qué es y qué no es aprendizaje cooperativo" en ( QVD RVI

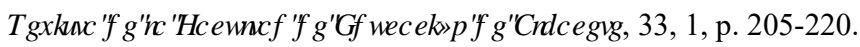

BREEZE, R. (2014). "Moodle glossary tasks for teaching legal English" en Eds. Elena Bárcena et al., / DQJ XDIHIRU USHUIFISXLSRVHLQWKHGJIWDOHD. Suiza: Springer International Publishing. P. 111-128.

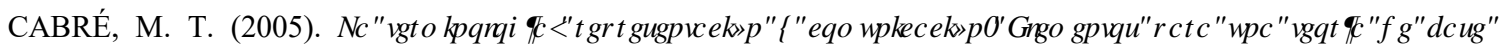
FRP XQIFDUMDI IRURVDUWFXGRV. Barcelona: Institut Universitari de Lingüística Aplicada.

CARVER, D. (1983). "Some propositions about ESP” en 7KH( 63L-RXUQD2, p. 131-137. 
GARCIA, J. Y LITZLER, M. F. (2015). "Current Perspectives in Teaching English for Specific Purposes" en 2 QRP i ]HQ 31, p. 38-51.

HUERTAS ABRIL, C. (2013). "La consulta a expertos como recurso documental en la traducción y revisión de textos especializados: recomendaciones para mejorar la intención comunicativa” en 6NRSRV,3, p. 75-87.

MORENO-PÉREZ, L. (2016). "El software de análisis de corpus aplicado a la docencia de LFE: propuesta metodológica” en ( QFXHQMR, 25, p. 62-75.

MORENO-PÉREZ, L. y LÓPEZ-ARROYO, B. (en prensa). “Atypical corpus-based tools to the rescue: how a writing genera-tor can help translators adapt to the demands of the market" en Calzada Pérez, M. y Laviosa, S. 5 HOH Ly QFUWFD HQCRVHMWGRVGHWDXFFIY QEDMDRVHQFRLSXVIO RQ7, 13.

MUÑOZ-MIQUEL, A. (2015). "El desarrollo de la competencia traductora a través de la socialización con el experto en la materia: una experiencia didáctica” en 7KH-RXLQDORIL6SHFDOMGT WOQDURQ 23, p. 333-351.

POSPISILOVA, L., BEZDICKOVA, Z., y CIBEROVA, D. (2011). "English for science using LMS Moodle” en 10 UK

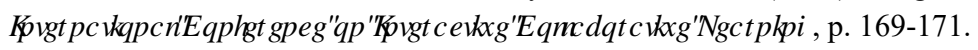

RATZ, S. (2016). "Vocabulary Learning with the Moodle Glossary Tool: A Case Study" en - RXLQDORIЗ HUSHFWMHIIQ

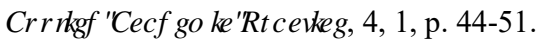

RODRIGUEZ PIÑERO, A.I., GARCIA ANTUÑA, M. (2009). "Lenguas de especialidad y lenguas para fines específicos: precisiones conceptuales y terminológicas e implicaciones didácticas” en Vera, A. y Martínez, I. (Eds.), ( 0 HSDKROHQFRQMU LRVHSHFIIFRVIHQMKDQJDHIQYHMWDFIYQ. Comillas: Fundación Comillas. P. 907-932.

RODRÍGUEZ-TAPIA, S. (2016). “Clasificación cuantitativa de los textos según su grado de especialidad: parámetros para la elaboración de los indices de densidad terminológica y de reformulación de un corpus sobre insuficiencia cardíaca" en \$QXDURIGH( WXGRV) IRQPJIFRV, XXXIX, p. 227-250.

SEGHIRI, M. (2013). "Creating a bilingual glossary (English-Spanish) based on Moodle for the teaching and learning of Scientific and Technical Translation" en Rivas-Carmona, M. M. y Balbuena Torezano, M. C. $\&$ \&QXWOQ\$ VSHWRI 7UDQDOMRQ Tübingen: Gunter Narr. P. 357-372. 